\title{
Bibliographie et filmographie de Bernard Juillerat
}

Isabelle Leblic

\section{OpenEdition}

Journals

Édition électronique

URL : http://journals.openedition.org/jso/6131

DOI : 10.4000/jso.6131

ISSN : $1760-7256$

Éditeur

Société des océanistes

Édition imprimée

Date de publication : 15 décembre 2010

Pagination : 21-28

ISBN : 978-2-85430-027-7

ISSN : 0300-953x

Référence électronique

Isabelle Leblic, «Bibliographie et filmographie de Bernard Juillerat », Journal de la Société des

Océanistes [En ligne], 130-131 | 2010, mis en ligne le 15 décembre 2010, consulté le 09 juin 2020.

URL : http://journals.openedition.org/jso/6131; DOI : https://doi.org/10.4000/jso.6131

(C) Tous droits réservés 


\title{
Bibliographie et filmographie de Bernard Juillerat
}

\author{
Établie par Isabelle Leblic*
}

Pour plus de clarté, cette bibliographie est présentée en décomposant l'ensemble des références de Bernard Juillerat, à savoir :

- les huit ouvrages et les trois directions d'ouvrages - en présentant les références des comptes rendus dont ils ont fait l'objet -,

- les cinquante-deux articles dans les revues,

- les quinze chapitres d'ouvrages

- et les quarante-huit comptes rendus de lecture.

Nous avons cité également les références des trois films réalisés par Bernard.

\section{Ouvrages}

Juillerat Bernard, 1969. Structures lignagères et règles matrimoniales chez les Mouktété (NordCameroun), Lausanne, Société suisse des sciences humaines, $281 \mathrm{p}$., publication de la thèse de $3^{\mathrm{e}}$ cycle de Lettres (Paris, 1966).

JuILLERAT Bernard, 1971. Les bases de l'organisation sociale chez les Mouktélé (Nord-Cameroun) : structures lignagères et mariage, Paris, Institut d'ethnologie, musée de l'Homme, avec le concours du CNRS, mémoires de l'Institut d'ethnologie 8, 271 p., publication de la thèse de $3^{\text {e }}$ cycle d'Ethnologie (Paris, 1969).

Burnham Philip, 1972. Reviewed work: Les bases de l'organisation sociale chez les Mouktélé (NordCameroun) : structures lignagères et mariage, by Bernard Juillerat, Man, New Series 7, 4, pp. 663-664.

Meunier Roger, 1973. C.R. de Bernard Juillerat, Les bases de l'organisation sociale chez les Mouktélé (Nord-Cameroun). Structures lignagères et mariage, Cahiers d'Études africaines 13, 49, p. 166.

Collard Chantal, 1973. C.R. de B. Juillerat, Les bases de l'organisation sociale chez les Mouktélé (NordCameroun). Structures lignagères et mariage, L'Homme 13, 1-2, pp. 241-243.
BraIN Robert, 1974. Reviewed work: Les bases de l'organisation sociale chez les Mouktélé (Nord-Cameroun) : Structures lignagères et mariage, by Bernard Juillerat, Africa: Journal of the International African Institute 44, 3, pp. 303-304.

JuILlERAt Bernard, 1984. The "bow" and the "skirt": the couple between masculine ideology and cultural fantasy in Yafar society (West Sepik), Basel, Wenner-Gren Foundation for Anthropological Research.

JuILlERAT Bernard, 1986. Les Enfants du sang: société, reproduction et imaginaire en NouvelleGuinée, Paris, Éditions de la maison des sciences de l'homme, $568 \mathrm{p}$.

Babadzan Alain, 1989. C.R. de B. Juillerat, Les enfants du sang: société, reproduction et imaginaire en Nouvelle-Guinée, L'Homme 29, 110, pp. 170-172.

Gell Alfred, 1989. Reviewed work: Les enfants du sang : société, Reproduction et imaginaire en NouvelleGuinée. by Bernard Juillerat, Man, New Series 24, 1, pp. 178-179.

Godin Patrice, 1990. Reviewed work: Les Enfants du sang : société, reproduction et imaginaire en NouvelleGuinée, by Bernard Juillerat, Archives de sciences sociales des religions 70, pp. 279-280.

Juillerat Bernard, 1991. Edipe chasseur : une mythologie du sujet en Nouvelle-Guinée, préface d'André Green, Paris, Presses universitaires de France, 292 p.

HiROYUKi Kurita, 1996. Reviewed work: CEdipe chasseur. Une mythologie du sujet en Nouvelle-Guinée [CEdipe the Hunter: A Mythology of the Subject in New Guinea ], by Bernard Juillerat, Asian Folklore Studies, 55, 2, pp. 389-390.

MAîTRE Jacques, 1991. Reviewed work(s): EEdipe chasseur. Une mythologie du sujet en Nouvelle-Guinée, by Bernard Juillerat, Archives de sciences sociales des religions 76, p. 269.

SCHWImmer Eric, 1994. Reviewed work: Edipe chasseur: Une mythologie du sujet en Nouvelle-Guinée, by Bernard Juillerat, American Ethnologist 21, 4, pp. 10261027.

* Ethnologue, CNRS LaCiTo, Villejuif, leblic@vjf.cnrs.fr 
JuILlerat Bernard, 1993. La révocation des Tambaran: les Banaro et Richard Thurnwald revisités, Paris, CNRs éd., CNRS ethnologie, 231 p.

Peltier Philippe, 1995. C.R. de B. Juillerat, La révocation des Tambaran. Les Banaro et Richard Thurnwald revisités, L'Homme 35, 133, pp. 158-161.

TuZIN Donald, 1994. Reviewed work: La révocation des Tambaran: Les Banaros et Richard Thurnwald revisités, by Bernard Juillerat, Man 29, 2, New Series, pp. 516-517.

JuILLERAT Bernard, 1995. L'avènement du père : rite, représentation, fantasme dans un culte mélanésie, Paris, CNRs éd., Éditions de la maison des sciences de l'homme, Chemins de l'ethnologie, 289 p.

BRUTTI Lorenzo, 1997. Reviewed work: L'avènement $d u$ père : rite, représentation, fantasme, dans un culte mélanésien, by Bernard Juillerat, American Ethnologist 24, 2, p. 504.

Simonis Yvan, 1997. C.R. de Bernard Juillerat, L'avènement du père. Rite, représentation, fantasme dans un culte mélanésien (Coll. Chemins de l'ethnologie, Paris, CNRS-Éditions et Éditions de la maison des sciences de l'homme, 1995, 290 p., bibliogr., gloss., cartes, tabl., fig., index), Anthropologie et Sociétés 21, 1, pp. 121-122.

STRATHERn Andrew, 1997. Reviewed work: L'avènement du père: rite, représentation, fantasme dans un culte mélanesien, by Bernard Juillerat, The Journal of the Royal Anthropological Institute 3, 4, pp. 821-822.

JuILleRAT Bernard, 1996. Children of blood: society, reproduction and cosmology in New Guinea, traduction de Nora Scott, New York, Oxford, Berg.

RobBins Joel, 1998. Reviewed work(s): Children of the Blood: Society, Reproduction and Cosmology in New Guinea by Bernard Juillerat, Anthropological Quarterly 71, 2, pp. 89-98.

STRATHERn Andrew, 1998. Reviewed work(s): Children of the Blood, by Bernard Juillerat (traduction de Nora Scott), American Anthropologist 100, 2, New Series, pp. $569-570$

Martin Keir, 2002. Reviewed work(s): Children of the Blood: Society, Reproduction and Cosmology in New Guinea, by Bernard Juillerat, The Journal of the Royal Anthropological Institute 8, 4, pp. 792-793.

Juillerat Bernard, 2001. Penser l'imaginaire : essais d'anthropologie psychanalytique, Lausanne, Payot, Anthropologie, $320 \mathrm{p}$.

NAePels Michel, 2002. C.R. de Bernard Juillerat, Penser l'imaginaire. Essais d'anthropologie psychanalytique, L'Homme 164, pp. 195-196.

Crapanzano Vincent, 2004. Reviewed work(s): Penser l'imaginaire: Essais d'anthropologie psychanalytique, by Bernard Juillerat, Current Anthropology 45, 1, Identity, Temporality, and Moral Geographies, pp. 127-128.

LÉzé Samuel, 2004. C.R. de Bernard Juillerat, Penser l'imaginaire. Essai d'anthropologie psychanalytique (Éditions Payot, Lausanne, 2001, 309 p., bibliogr. Index), Anthropologie et Sociétés 28, 3, pp. 225-226.

\section{Direction d'ouvrages}

JUILLERAT Bernard (ed.), 1992. Shooting the sun: ritual and meaning in West Sepik, Washington and Lon- don, Smithsonian Institution Press, Smithsonian series in ethnographic inquiry, in cooperation with the ministère des Affaires étrangères-Paris, $310 \mathrm{p}$.

ERRINGTON Frederick, 1995. Reviewed work: Shooting the Sun: Ritual and Meaning in West Sepik, by Bernard Juillerat, American Ethnologist 22, 2, pp. 436-437.

WeInER James F., 1995. Reviewed work(s): Shooting the Sun: Ritual and Meaning in West Sepik, by Bernard Juillerat, The Journal of the Royal Anthropological Institute 1, 2, pp. 442-443.

Pradelles de Latour Charles-Henry, 1997. C.R. de B. Juillerat, Shooting the sun. Ritual and meaning in West Sepik, L'Homme 37, 142, pp. 164-166.

Juillerat Bernard et Monique Jeudy-Ballini (eds), 2002. People and things: social mediations in Oceania, Durham, NC, Carolina Academic Press, ix, $363 \mathrm{p}$.

Lederman Rena, 2004. Review People and Things: Social Mediations in Oceania, by Jeudy-Ballini Monique et Bernard Juillerat (éds), The contemporary Pacific 16, 2, pp. 452-454.

NAEPEls Michel, 2005. C.R. de M. Jeudy-Ballini et B. Juillerat (eds), People and Things: Social Mediations in Oceania, L'Homme 173, pp. 205-206.

Juillerat Bernard, Patrice Bidou et Jacques GAliNIER (éds), 2005. Psychanalyse et anthropologie. Regards croisés, Paris, Éditions de l'EHEss, collections Cahiers de l'Homme 37, 230 p.

LÉzÉ Samuel, 2005. C.R. de Patrice Bidou, Jacques Galinier et Bernard Juillerat (dir.), Anthropologie et psychanalyse. Regards croisés (Paris, Éditions de l'EHESS, collection Cahiers de l'Homme, 2005, 228 p.), Anthropologie et Sociétés 31, 2, pp. 312-313.

SÈre Bénédicte, 2006. C.R. de Patrice Bidou, Jacques Galinier, Bernard Juillerat (éds), Anthropologie et psychanalyse. Regards croisés, Archives de sciences sociales des religions 134, pp. 157-158.

Mestre Claire, 2008. C.R. de Patrice Bidou, Jacques Galinier, Bernard Juillerat (éds), Anthropologie et psychanalyse. Regards croisés, L'Homme 185-186, pp. 495-497.

\section{Articles dans des revues}

JuILlERAT Bernard, 1968. Note sur les origines d'une ethnie " kirdi »: les Mouktélé (Nord-Cameroun), Journal de la société des africanistes 38, 2, pp. 95112.

JuILlERAT Bernard, 1968. Règles et rites matrimoniaux chez les Mouktélé du Nord-Cameroun, Journal de la société des africanistes 38, 2, pp. 113-136.

JuILlERAT Bernard, 1970. Note sur trois relations de parenté chez les Mouktélé (Nord-Cameroun), Cahiers d'études africaines 10, 39, pp. 469-478.

JuILlERAT Bernard, 1971-1972. Ethnographie des Amanab : aperçu sur la culture d'une ethnie du Sépik occidental (Nouvelle-Guinée), Bulletin de la Société suisse d'Anthropologie et d'Ethnologie, $48^{\mathrm{e}}$ année, pp. 105-129. 
JuILlerat Bernard, 1972. Communauté et tenure foncière dans trois villages du Sepik occidental, Journal de la Société des Océanistes 28, 35, pp. 103-140.

JuILlERAT Bernard, 1973. Brève visite d'une maison commune chez les Biami (Nouvelle-Guinée), Anthropos. Internationale zeitschrift für Volker- und sprachenkunde 68, pp. 777-784.

Juillerat Bernard, 1975. Les Amanab du HautSepik. Compte rendu de mission, L'Homme 15, 1, pp. 103-108.

JuILlerat Bernard, 1975. Transe et langage en Nouvelle-Guinée (1 ${ }^{\text {ère }}$ partie). I. La possession médiumnique chez les Amaba, Journal de la Société des Océanistes 31, 47, pp. 187-212.

JuILlERAT Bernard, 1975. Transe et langage en Nouvelle-Guinée ( $2^{\text {ème }}$ partie). II. Du symptôme au rite, Journal de la Société des Océanistes 31, 49, pp. 379-397.

Juillerat Bernard, 1975. Dessins de sol du HautSepik, Objets et mondes 15, 4, pp. 385-396.

JuILLERAT Bernard, 1977. « Folie », possession et chamanisme en Nouvelle-Guinée. Introduction, Journal de la Société des Océanistes 33, 56-57, pp. 117 121.

Juillerat Bernard, 1977. Terminologie de parenté iafar (Nouvelle-Guinée). Étude formelle d'un système dakota-iroquois, L'Homme 17, 4, pp. 5-33.

JuILlERAT Bernard, 1979. En route pour les plantations. Récit recueilli et introduit par Bernard Juillerat, Journal de la Société des Océanistes 35, 64, pp. 209-212.

JuILlerat Bernard, 1980. À propos de l'initiation Gnau. Compte rendu de l'ouvrage de Gilbert Lewis : Days if shining red. An essay in understanding ritual (Cambridge University Press, 1980, 233 p.), Journal de la Société des Océanistes 36, 68, pp. 227-232.

Juillerat Bernard, Andrew Strathern, Ron BrunTON and Aflerd Gell, 1980. Order or disorder in Melanesian religions?, Man, New Series 15, 4, pp. 732-737.

JuILleRAT Bernard, 1980. Humour et transgression dans la littérature orale d'une société de NouvelleGuinée, Cahiers de littérature orale 8, pp. 125-149.

JuILLERAT Bernard, 1981. Organisation dualiste et complémentarité sexuelle dans le Sepik occidental, L'Homme 21, 2, pp. 5-38.

Juillerat Bernard, 1982. Note sur les rapports de production dans l'horticulture-arboriculture yafar (Nouvelle-Guinée), Journal d'agriculture traditionnelle et de botanique appliquée 29, 3-4, pp. 285-293.

JuILlerat Bernard, 1983. L'essartage chez les Yafar (Nouvelle-Guinée), Journal d'agriculture traditionnelle et de botanique appliquée 30, 1, pp. 3-33.

JuILleRAT Bernard, 1984. D'Acorus à Zingiber : taxinomie et usages des plantes cultivées chez les Yafar de Nouvelle-Guinée, Journal d'agriculture traditionnelle et de botanique appliquée 31, 1-2, pp. 3-31.
JuILlERAT Bernard, 1984. Culture et exploitation du palmier-sagoutier dans les Border Mountains (Nouvelle-Guinée), Techniques \& culture 3, pp. 4364.

Juillerat Bernard, 1984. La mort d'Arnold Ap et la destruction des cultures de Nouvelle-Guinée occidentale, Journal de la Société des Océanistes 40, 78, pp. 103-106.

Juillerat Bernard, 1985. Échos Papouasie occidentale, Bulletin de l'AFA 20, Paris, maison des sciences de l'homme, pp. 103-109.

JuILlERAT Bernard, 1987. I colori del sacro : danza, musica e maschere di un rito suggestivo e ricco du significati, Prometeo. Rivista trimestrale di scienze e storia 19 , pp. 50-53.

JuILlERAT Bernard, 1987. Réponses à l'enquête : Qui a peur de Laura Makarius ?, Gradhiva 3, pp. 60-62.

JuILlERAT Bernard, 1988. «Une odeur d'homme » : évolutionnisme mélanésien et mythologie anthropologique à propos du matriarcat, Diogène 144, pp. 65-91.

JuILleRAT Bernard, 1988. «An odor of man»: Melanesian evolutionism, anthropological mythology and matriarchy, Diogène 144, pp. 65-91.

JuILlerat Bernard and Simon HARrison, 1990. Ritual complementarity and Political rivalry in the Sepik, Man 25, 2, New Series, pp. 337-340.

JuILLERAT Bernard, 1992. L'univers dans un hameau : cosmologie et histoire chez les Yafar (Papouasie Nouvelle-Guinée), Études rurales 127-128, pp. 159176.

JuILLERAT Bernard, 1993. L'air, le feu, le son : fabrication et usage des trompettes et des tambours dans les Border Mountains (Papua New Guinea), Baessler-Archiv 41, pp. 413-444.

JuILlerat Bernard, 1993. Des fantasmes originaires aux symboles culturels : médiations et seuils, Revue française de psychanalyse 3, pp. 713-731.

JuILlERAT Bernard, 1993. Richard Thurnwald et la Mélanésie: réciprocités, hiérarchies, évolution, Gradhiva 14, pp. 15-40.

JuILlerAt Bernard, 1995. Du roman familial à la honte d'engendrer, L'Homme 35, 135, pp. 87-108.

Juillerat Bernard, 1995. Entre Freud et Yung : le mythe. La dissidence jungienne comme point de rupture épistémologique, Gradhiva 18, pp. 27-45.

Juillerat Bernard, 1996. Air, fire, sound: the construction and use of trumpets and drums in the Border Mountains (Papua New Guinea), Kulele 2, pp. 1-30.

Juillerat Bernard, 1996. Anthropologie/Psychanalyse: les handicaps d'un dialogue, Journal des anthropologues 64-65, pp. 19-31.

JuILLERAT Bernard, 1997. L'envers du don : du désir à l'interdit. Représentations de l'échange et structure œdipienne dans une société mélanésienne, Social Anthropology 5, 1, pp. 11-20. 
JuILlerat Bernard, 1997. Yangis, Lacan and caducity: a reply to James Weiner, Social analysis. Journal of cultural and social practice 41, 2, pp. 35-54.

JuILlERAT Bernard, 1998. Totémisme duel, totémisme pluriel : un exemple de Nouvelle-Guinée, Systèmes de pensée en Afrique noire 15, pp. 109-125.

JuILLERAT Bernard, 1998. L'insoutenable légèreté de l'ego masculin ou la vengeance du casoar, L'Homme 38, 146, pp. 207-217.

JuILleRAT Bernard, Jacques GALINIER et Patrice BIDOU, 1999. Arguments, L'Homme 39, 149, pp. 7-24.

JuILLERAT Bernard, 1999. La mort yafar. Les métamorphoses du sujet dans une culture mélanésienne, L'Homme 39, 149, pp. 63-72.

JuILleRAT Bernard, 1999. Séparation, retour, permanence. Le lien maternel dans le rite naven des Iatmul, L'Homme 39, 151, pp. 151-180.

JuILLERAT Bernard, 1999. L'histoire en morceaux, L'Homme 39, 152, pp. 163-172.

JuILleRAT Bernard, 2000. Do the Bánaro really exist? Going back after Richard Thurwald, Oceania 71, pp. 46-66.

JUILLERAT Bernard, 2001. L'atome de parenté est-il soluble dans la psychanalyse ?, Topique 75, pp. 81103.

JuILlERAT Bernard, 2001. Mère interdite, mère promise ou la prophétie des origines, Perspective Psy $40, \mathrm{n}^{\circ}$ spécial, publication du colloque de l'association ERIE : Aspects de la notion de temps dans la prise en charge en psychiatrie, pp. 40-45.

Juillerat Bernard, 2002. Tapir chamane et Grand collant céleste. Une figure clivée de la mythologie tatuyo (Nord-Ouest amazonien), L'Homme 164, pp. $125-134$.

JuILlERAT Bernard, 2002. Réponse de Bernard Juillerat, Anthropologie et Sociétés 26, 2-3, pp. 256-259.

JuILLERAT Bernard, 2003. Un mythe est-il un rêve collectif ?, Topique 84, pp. 33-42.

JUILLERAT Bernard, 2004. Reconnaissance de l'inconscient. Regards anthropologiques sur l'œuvre d'André Green, L'Homme 169, pp. 195-206.

Juillerat Bernard, 2004. Psyche, culture and society: book review forum on Bernard Juillerat's « Penser l'imaginaire. Essais d'anthropologie psychanalytique », Journal of ritual studies 18, 1, pp. 158-166.

JuILleRAT Bernard, 2004. À l'origine des techniques, l'interdit? Escales océaniennes, Techniques \& culture 43-44 : Mythes. L'origine des manières de faire, pp. 3-23

JuILlERAT Bernard, 2005. Separation, return, permanence: the maternal bond in the naven ritual of the Iatmul, Journal of ritual studies 19, 2, pp. 71-87.

\section{Chapitres d'ouvrages collectifs}

JuILLERAT Bernard, 1974. Éléments d'ethno-histoire des Mukteke et du Mandara septentrional, in
Contribution de la recherche ethnologique à l'histoire des civilisations du Cameroun / The contribution of ethnological research to the history of Cameroon Cultures, Paris, CNRs, Colloques internationaux du CNRS 551, vol. 1, pp. 205-208.

JuILlERAT Bernard, 1978. Techniques et sociologie de la couleur chez les Iafar (Nouvelle-Guinée), in Serge Tornay (éd.), Voir et nommer les couleurs, Nanterre, université de Paris X, Publications du laboratoire d'ethnologie et de sociologie comparative, pp. 477495.

JUILLERAT Bernard, 1978. Vie et mort dans le symbolisme Iafar des couleurs (Nouvelle-Guinée), in Serge Tornay (éd.), Voir et nommer les couleurs, Nanterre, université de Paris X, Publications du laboratoire d'ethnologie et de sociologie comparative, pp. 497523.

JuILLERAT Bernard, 1983. Les modalités de l'échange chez les Eri de Nouvelle-Guinée, in Séminaire sur les structures d'habitat, Paris, Collège de France, Chaire de préhistoire, pp. 78-87.

JuILLERAT Bernard, 1990. Male ideology and cultural fantasy in Yafar society, in Nancy Lutkehaus et al. (eds), Sepik Heritage : tradition and change in Papua New Guinea, Durham, Carolina Academic Press, pp. 380-384.

JuILleRAT Bernard, 1991. Complementarity and riva1ry: two contradictory principles in Yafar society, in Maurice Godelier and Marilyn Strathern (eds), Big men and great men: personifications of power in Melanesia, Cambridge-New York-Melbourne, Cambridge University press et Paris, Éditions de la maison des sciences de l'homme, pp. 130-141.

JUILLERAT Bernard, 1992. The mother's brother is the breast: Incest and Its Prohibition in the Yafar Yangis, in Bernard Juillerat (ed.), Shooting the sun: ritual and meaning in West Sepik, WashingtonLondon, Smithsonian Institution Press, Smithsonian series in ethnographic inquiry, pp. 20-124.

JuILLERAT Bernard, 1995. L'avènement du rite: ethnopsychanalyse d'un culte mélanésien, in B. Juillerat (éd.), L'avènement du père : rite, représentation, fantasme dans un culte mélanésien, Paris, CNRs éd., Éditions de la maison des sciences de l'homme, Chemins de l'ethnologie.

JuILlERAT Bernard, 1996. Le sagou dans une société de Papouasie Nouvelle-Guinée, in Marie-Claire Bataille-Benguigui et Françoise Cousin, Cuisines : reflets des sociétés, Paris, éditions Sépia, musée de l'Homme, pp. 45-55.

JUILlERAT Bernard, 1997. « My poor dwellers » (Yafar 1970-1995), in Françoise DouaireMarsaudon et Serge Tcherkézoff, Le Pacifique-Sud aujourd'hui : identités et transformations culturelles, Paris, CNRs Éditions, pp. 59-76.

JuILleRAT Bernard, 1999. Rationalité technique et logique symbolique. De l'essartage au mythe en Nouvelle-Guinée, in Jean-Luc Jamard, Annie Montigny \& François-René Picon (éds), Dans le sillage des techniques : Hommage à Robert Cresswell, Paris, L'Harmattan, pp. 195-224. 
JuILlerat Bernard, 2000. Et 1'homme créa la femme. Mythes et non-mythes de Nouvelle-Guinée, in Margarita Xanthakou, Jean-Luc Jamard et Emmanuel Terray (éds), En substances : textes pour Françoise Héritier, Paris, Fayard, pp. 397-409.

JuILlERAT Bernard, 2002. Le rite comme acte et comme représentation : de l'inconscient à l'espace transitionnel, in César Botella (éd.), Penser les limites : écrits en l'honneur d'André Green, Paris, Delachaux et Niestlé, pp. 44-51.

Juillerat Bernard, 2002. The Other Side of the Gift: From Desire to Taboo: Representations of Exchange and Oedipal Symbolism among the Yafar, Papua New Guinea, in Monique JeudyBallini \& Bernard Juillerat (eds), People and Things: Social Mediations in Oceania, Durham, NC, Carolina Academic Press, pp. 157-183.

Juillerat Bernard, Patrice Bidou et Jacques GaliNIER, 2005. Présentation, in Bernard Juillerat, Patrice Bidou et Jacques Galinier (éds), Anthropologie et psychanalyse. Regards croisés, Paris, éditions de l'EHESS, pp. 7-25.

JuILleRAT Bernard, 2005. Plaisir et réalité : la notion de « cargo » revisitée, in Bernard Juillerat, Patrice Bidou et Jacques Galinier (éds), Anthropologie et psychanalyse. Regards croisés, Paris, éditions de l'EHESS, pp. 81-97.

JuILleRAT Bernard, 2008. « My poor border dwellers » (Yafar 1970-1995), in Serge Tcherkézoff et Françoise Douaire-Marsaudon, The Changing South Pacific, ANU E Press, pp. 43-62.

\section{Comptes rendus d'ouvrages}

Juillerat Bernard, 1972. C.R. de Jacqueline M.C. Thomas et Lucien Bernot (éds), Langages et techniques, nature et société (Paris, Klincksieck, 1972, 2 vol., 400 p. et 416 p.), Journal de la Société des Océanistes 28, 36, pp. 316-317.

JuILlerat Bernard, 1974. C.R. de J.-G. Gauthier, Les Fali de Ngoutchoumi, montagnards du NordCameroun, L'Homme 14, 1, pp. 89-91.

Juillerat Bernard, 1974. C.R. de Tetaria Charles, Réflexions épidémiologiques à propos de l'évolution de la filariose à Tahiti après vingt-cinq ans de lutte antifilarienne (Rennes, 1974, 142, XXVII p., thèse pour le doctorat en médecine), Journal de la Société des Océanistes 30, 45, p. 315.

Juillerat Bernard, 1974. C.R. de Whiteman J., Chimbu family relationships in Port-Moresby (PortMoresby and Canberra, New Guinea Research Unit, Australian University, 1973, New Guinea Bulletin 52), Journal de la Société des Océanistes 30, 45, pp. 315-316.

Juillerat Bernard, 1975. C.R. de L. Morauta, Beyond the village. Local politics in Madang, Papua New Guinea, L'Homme 15, 2, pp. 140-142.
Juillerat Bernard, 1976. C.R. de K.-F. Koch, War and peace in Jalémó. The management of conflict in Highland New Guinea, L'Homme 16, 1, pp. 175-176.

Juillerat Bernard, 1976. C.R. de A. Montagu, Corning into being among Australian Aborigines. A study of the procreative beliefs of the native tribes of Australia, L'Homme 16, 1, pp. 176-178.

JuILlerat Bernard, 1976. C.R. de F. Barth, Ritual and knoledge among the Baktaman of New Guinea, L'Homme 16, 2, p. 190.

Juillerat Bernard, 1977. C.R. de Delaby Laurence, Chamanes toungouses (Études mongoles 7, 1976, 243 p.) et de Voyages chamaniques (L'ethnographie $\mathrm{n}^{\circ}$ spécial 74-75, 1977, 271 p.), Journal de la Société des Océanistes 33, 56-57, p. 224.

JuILlerat Bernard, 1977. C.R. de McGregor Donald, The fish and the cross (Hamilton N.-Z., 1975, édité par l'auteur), Journal de la Société des Océanistes 33, 56-57, pp. 224-225.

Juillerat Bernard, 1977. C.R. de John Kolia, The history of the Balawaia, de Georg F. Vicedom, Myths and legends from Mount Hagen et de R.P. Karl Hess, Baining legends, Journal de la Société des Océanistes 33, 56-57, pp. 228-229.

Juillerat Bernard, 1977. C.R. de A. Gell, Metamorphosis of the Cassowaries. Umeda Society, Language and ritual, L'Homme 17, 2, pp. 165-166.

JuILlERAT Bernard, 1978. C.R. de F.E. Williams, «The Vailala madness» and other essays, L'Homme 18, 1, pp. 221-222.

Juillerat Bernard, 1978. C.R. de C.R. Hallpike, Bloodshed and vengeance in the Papuan Moutains. The generation of conflict in Tauade society, L'Homme 18,1, pp. 222-224.

Juillerat Bernard, 1978. C.R. de Daniel de Coppet et Hugo Zemp, 'Aréaré un peuple mélanésien et sa musique (Paris, éditions du Seuil, 1978, 127 p. et disque encarté), Journal de la Société des Océanistes 34, 60, pp. 139-140.

Juillerat Bernard, 1979. C.R. de David R. Moore, Islanders and Aborigines at Cape York. An ethnographic renconstruction based on the 1848-1850 'Rattlesnake' Journals of O.W. Brierly and information he obtained from Barbara Thompson (Canberra, Australian Institute of Aboriginal Studies, Humanities Press Inc., New Jersey (USA), 1979, xii + 340 p.), Journal de la Société des Océanistes 35, 63, pp. 150151.

JuILlerat Bernard, 1979. C.R. de Hermione Frankel, Canoes of Walamo (Institute of Papua New Guinea Studies, 1978, 60 p.), Journal de la Société des Océanistes 35, 63, pp. 149-150.

Juillerat Bernard, 1979. C.R. de Michele Stephen, Cargo cult hysteria: symptom of despair or technique of ecstasy? (Research Centre for Southwest Pacific Studies, La Trobe University, Occasional Paper 1, 1977, 16 p.), Journal de la Société des Océanistes 35, 63, p. 153. 
Juillerat Bernard, 1979. C.R. de Donald F. Tuzin, The Dahita Arapesh. Dimensions of unity (University of California Press, 1976, 376 p.), Journal de la Société des Océanistes 35, 63, pp. 153-154.

Juillerat Bernard, 1979. C.R. de Markus Schindlbeck, Sago bei den Sawos (Mittelsepik, Papua New Guinea). Untersuchung über die Bedeutung von Sago in Wirtschaft, Socialordnung und Religion (Basel, Ethnologisches Seminar der Universität und Museum für Völkerkunde, Basel, 19, 1980, 566 p.), Journal de la Société des Océanistes 35, 64, pp. 215216.

JuILlerat Bernard, 1980. C.R. de Donald Tuzin, The voice of the Tambaran. Truth and illusion in Ilahita Arapesh Religion (Berkeley, Los Angeles, London, University of California Press, 355 p.), Journal de la Société des Océanistes 36, 69, pp. 318-319.

Juillerat Bernard, 1981. C.R. de Antje et Heinz Kelm, Sago und Schwein. Ethnologie von Kwieftin und Abrau in Nordost-Neuguinea (Studien zur Kulturkunde 51, Wiesbaden, Franz Steiner Verlag, 1980, 397 p.), Journal de la Société des Océanistes 37, 72-73, pp. 312-313.

Juillerat Bernard, 1988. C.R. de M.S. Mosko, Quadripartite structures. Categories, relations, and homologies in bush Mekeo culture, L'Homme 28, 105, pp. 154-155.

JuILlerat Bernard, 1988. C.R. de K.E. Read, Return to the High Valley. Coming full circle, L'Homme 28, 105, pp. 155-156.

Juillerat Bernard, 1989. C.R. de F. Barth, Cosmologies in the Making. A new generation approach to cultural variation in Inner New Guinea, L'Homme 29, 111, pp. 287-291.

Juillerat Bernard, 1990. C.R. de B.M. Knauft, Good company and violence. Sorcery and social action in a Lowland New Guinea society, L'Homme 30, 115, pp. 172-174.

Juillerat Bernard, 1991. C.R. de G. Herdt et R. Stoller, Intimate communications. Erotics and the study of culture, L'Homme 31, 120, pp. 113-115.

Juillerat Bernard, 1991. C.R. de M. Melk-Koch, Auf der Suche nach der menschlischen Gesellschaft: Richard Thurnwald, L'Homme 31, 120, pp. 115-118.

Juillerat Bernard, 1994. C.R. de N. McDowell, The Mundugumor. From field notes of Margaret Mead and Reo Fortune, L'Homme 34, 131, pp. 189-191.

JuILlerat Bernard, 1994. C.R. de G. Gillison, Between culture and fantasy. A New Guinea Highlands mythology, L'Homme 34, 131, pp. 209-212.

Juillerat Bernard, 1994. C.R. de D.B. Gewertz et F.K. Errington, Twisted histories, altered contexts. Representing the Chambri in a world system, L'Homme 34, 131, pp. 191-193.

Juillerat Bernard, 1995. C.R. de J. W. Schoorl, Culture and Change among the Muyu, L'Homme 36, 140, pp. 161-162.

Juillerat Bernard, 1997. C.R. de J.C. Goodale, To sing with pigs is human. The concept of person in Papua New Guinea, L'Homme 37, 141, pp. 195-196.
Juillerat Bernard, 1997. C.R. de N.C. Lutkehaus, Zaria's fire? Engendered moments in Manam ethnography, L'Homme 37, 141, pp. 197-198.

Juillerat Bernard, 1997. C.R. de S. Ohnemus, Zur Kultur des Admiralitäts-Insulaner in Melanesien. Die Sammlung Alfred Bühler im Museum für Völkerkunde Basel, L'Homme 37, 143, p. 233.

JuILlERAT Bernard, 1997. C.R. de L. Lindstrom, Cargo cult. Strange stories of desire from melanesian and beyond, L'Homme 37, 144, pp. 221-222.

Juillerat Bernard, 1998. C.R. de Brigitte Derlon, De mémoire et d'oubli. Anthropologie des objets malanggan de Nouvelle-Irlande, L'Homme 38, 147, pp. 295298.

Juillerat Bernard, 2000. C.R. d'Élisabeth Roudinesco, Pourquoi la psychanalyse? (Paris, Fayard, 198 p.), L'Homme 156, pp. 269-271.

Juillerat Bernard, 2001. C.R. de Alban Bensa \& Jean-Claude Rivierre (éds), Le Pacifique. Un monde épars. Introduction interdisciplinaire à l'étude de l'Océanie (Paris-Montréal, L'Harmattan, 1998, 214 p., bibl., fig., tabl., cartes (« Cahiers du Pacifique Sud contemporain »), L'Homme 158-159, pp. 483-484.

JuIllerat Bernard, 2002. C.R. de Donald Tuzin, Social Complexity in the Making. A Case Study among the Arapesh of New Guinea (London \& New York, Routledge, 2001, xii + 159 p., gloss., index, réf., fig., ph., cartes), L'Homme 161, pp. 295-296.

JuIllerat Bernard, 2002. C.R. de Eric Kline Silverman, Masculinity, Motherhood, and Mockery. Psychoanalyzing Culture and the Iatmul Naven Rite in New Guinea (Ann Arbor, The University of Michigan Press, 2001, xiii +245 p., réf., index, ill., cartes), L'Homme 164, pp. 196-199.

Juillerat Bernard, 2003. C.R. de Monod Becquelin \& Alain Breton, La " Guerre Rouge » ou une politique maya du sacré. Un carnaval tzeltal au Chiapas, Mexique (Paris, CNRs Éd., 2002, 367 p., bibl., gloss., ill., fig., cartes), L'Homme 167-168, pp. 368-370.

Juillerat Bernard, 2004. C.R. de Alfred Adler, Le Pouvoir et l'Interdit. Royauté et religion en Afrique noire: essais d'ethnologie comparative, L'Homme 169 , pp. 261-266.

JuILlerat Bernard, 2004. C.R. de Pierre-Loïc Pacaud, Un culte d'exhumation des morts à Madagascar : le Famadihana. Anthropologie psychanalytique, L'Homme 170, pp. 301-304.

Juillerat Bernard, 2004. C.R. de Marlène AlbertLlorca \& José Antonio González Alcantud (eds), Moros y Cristianos: representaciones del otro en las fiestas del Mediterráneo occidental, L'Homme 170, pp. 311-313.

Juillerat Bernard, 2005. C.R. de Monique JeudyBallini, L'art des échanges. Penser le lien social chez les Sulka (Lausanne, Payot, 2004, 348 p.), Gradhiva 2, pp. 149-151.

JuILlERAT Bernard, 2005. C.R. de Yoram Mouchenik, L'enfant vulnérable. Psychothérapie transculturelle 
en pays kanak (Nouvelle-Calédonie) (Paris, La Pensée sauvage, 2004, préfaces de Marie-Rose Moro et d'Alban Bensa, 255 p.), Gradhiva 2, pp. 151-152.

\section{Microfilm, microfiches}

JuILLERAT Bernard et musée national de l'Homme d'Ottawa, 1974. Objets du Haut-Sepik, NouvelleGuinée (Amanab et Kwomtari) : notes techniques et ethnographiques, s.1., 501 leaves.

Juillerat Bernard, 1975. Objets du Haut-Sepik, Nouvelle-Guinée (Amanab et Kwontari), Microfiches $n^{\circ}$ I 74099 117, Paris, Institut d'Ethnologie, Musée de l'Homme.

Juillerat Bernard, nd. Amanab-English lexions and test Amanab language of PNG, Melanesian manuscript series $\mathrm{n}^{\circ} 0142$ [microfiches].

\section{Traduction}

Madan Triloki Nath, 1990. À l'opposé du renoncement : perplexités de la vie quotidienne hindoue, trad. de l'anglais par Denise Paulme-Schaeffner et Bernard Juillerat, Paris, Éditions de la maison des sciences de l'homme, $214 \mathrm{p}$.

\section{Vidéographie}

Juillerat Bernard (réal. de), 1968. Matsam, Meudon, CNRS Audiovisuel, 1 cass.vidéo (vHS), 23 min., coul., SECAM, sonore, Français.

JuILlERAT Bernard (réal. de), 1973. Un Jardin à Iafar, Meudon, CNRS Audiovisuel, 1 vidéocassette (VHS), 1 h 28 mn, coul., 1/2 po, sonore, Français.

Juillerat Bernard (réal. de), 1981. Le Sang du sagou, Meudon, CNRs Audiovisuel (éd., distrib.), 1 cassette vidéo (VHS), 55 min, coul., SECAM, sonore, collection Sciences de l'homme et de la société, Français.

Owen Chris (Bernard Juillerat \& Alfred Gell, scientific advisors), 1983. The Red Bowman, Goroka, National Film Institute.

\section{In memoriam Bernard Juillerat}

AnONYme, 2006. Hommage à Bernard Juillerat, Journal de la Société des Océanistes 122-123, p. 224.

COIFFIER Christian, 2006. Bernard Juillerat. In memoriam, Gradhiva 4, p. 124.

GALINIER Jacques, 2007. Bernard Juillerat (19372006), L'Homme 181, pp. 203-208. 
